\section{Sport auf Rezept: Gehtraining bei Gefäßkranken}

\author{
In deutschen und internationalen Leitlinien zur peripheren \\ arteriellen Verschlusskrankheit besitzt der Gefäßsport mit \\ seinem Herzstück, dem strukturierten Gehtraining, einen \\ hohen Stellenwert. Doch wie sieht die Umsetzung in der Praxis \\ aus? Ein Angiologe, der seit 15 Jahren Gefäßtrainer ausbildet, \\ meint: „Es wird zu viel operiert und dilatiert und viel zu wenig \\ trainiert!" Ist das wirklich so?
}

Für Patienten mit Claudicatio intermittens $(\mathrm{CI})$ und peripherer arterieller Verschlusskrankheit (PAVK) in den FontaineStadien I und II ist strukturierter Gefäßsport empfehlenswert, soweit sie dazu in der Lage sind. Dies sagt in Deutschland die entsprechende S3-Leitline, die europäische Fachgesellschaft European Society of Cardiology (ESC) bezieht die gleiche Position. Das Training soll mindestens dreimal wöchentlich in Übungseinheiten von 30 bis 60 Minuten über einen Zeitraum von mindestens drei Monaten erfolgen; ideal wäre, es lebenslang fortzuführen, sofern sich die PAVK nicht verschlechtert.

\section{Studiendaten belegen Nutzen}

Grundlage der evidenzbasierten Empfehlungen mit dem Empfehlungsgrad A Level 1 sowohl der deutschen Fachgesellschaft DGA von 2009, der europäischen Fachgesellschaft ESC von 2012 als auch der amerikanischen Fachgesellschaft AHA/ACC (2005) bilden zahlreiche Studien, in denen nachgewiesen werden konnte, dass supervidiertes Gehtraining über mindestens 12 Wochen dreimal wöchentlich für 30-60 min die Gehstrecke signifikant verlängerte - je nach Arbeit zwischen $150 \%$ und $246 \%$ im Vergleich zum Ausgangswert. Darüber hinaus konnte nachgewiesen werden, dass regelmäßiges Gehtraining bei Patienten mit PAVK die Mortalität senkt (Circulation 2006, 114: 242-248, Circulation 2011, 123: 89-97, Circulation 2011, 123: 491-498). Somit ist neben einer lokalen Wirkung auf die Atherosklerose im Bereich der Beingefäße auch eine übergreifende Wirkung auf die kardiovaskuläre Mortalität zu verzeichnen.
Spazierengehen reicht nicht aus

Dr. med. Gesine Dörr, Fachärztin für Innere Medizin mit der Spezialisierung Kardiologie/Angiologie ist Chefärztin der

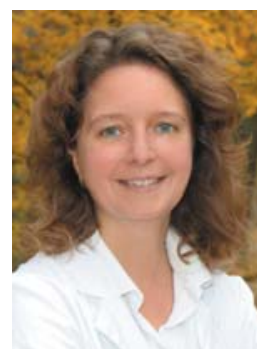
Wolletzsee-Klinik, einer Reha-Einrichtung der GLG Gesellschaft für Leben und Gesundheit $\mathrm{mbH}$ mit dem Schwerpunkt auf angiologische und neurologische $\mathrm{Re}$ habilitation. Die Landschaft um den malerischen Wolletzsee verlockt zu ausgedehnten Spaziergängen. „Nicht überwachtes, individuelles Gehen ist aber deutlich weniger effektiv als ein strukturiertes Trainingsprogramm“, betont Dörr. Es sei auch wichtig, dass die Trainingsintensität bis zum Belastungsschmerz ausgedehnt wird. „Wir vermitteln unseren Patienten daher, dass Gehtraining nicht etwa ,gemütliches Schlendern' bedeutet, sondern zügiges Gehen mit durchschnittlich 100 Schritten pro Minute, bis leichte Schmerzen zu spüren sind. Dann wird eine Pause eingelegt, bis diese abgeklungen sind. Dies wird mehrmals wiederholt. Wir stimmen das Pensum genau auf das Erkrankungsstadium und die Kondition unserer Patienten ab.“

„Bevor das eigentliche Training beginnen kann, vermitteln wir unseren Patienten die richtigen Fußbewegungen und $\mathrm{Ab}$ rolltechniken“, erläutert Sporttherapeut Christian Brückner, der Dörr bei der Ausarbeitung des Trainingsprogramms unterstützt. „Denn viele Patienten mit Durchblutungsstörungen haben sich Fehlhaltungen angewöhnt, um die
Dr. med. Gesine Dörr

Schmerzen beim Gehen zu verringern, diese behindern ein effektives Training." Auch die richtige Armhaltung sei für das Gehen sehr wichtig und werde geübt. Desweiteren helfen Kraft- und Koordinationsübungen, bisher wenig genutzte Beinmuskeln zu kräftigen. ,Je koordinierter die Muskelgruppen zusammenarbeiten, umso kraftsparender wird das Gehen“, erklärt Brückner. Auch Dehnungsübungen, „Muskelpflege“ genannt, gehören zum Gefäßsport dazu. Nach der Trainingsstunde wird die erreichte Strecke in das persönliche Therapieheft eingetragen. „Für die Patienten ist es eine große Motivation zu sehen, wie die sportliche Betätigung ihre Gehstrecke nach und nach verlängert", berichtet Brückner, der eine Zusatzausbildung zum Gefäßtrainer absolviert hat.

Doch die Verlängerung der Gehstrecke ist nicht der einzige Erfolg, den die Patienten verbuchen können. „Untersuchungen haben gezeigt, dass regelmäßiges Gehtraining das Risiko senkt, an einer Durchblutungsstörungen frühzeitig zu sterben“, erklärt Dörr. Dr. med. Clemens Fahrig,

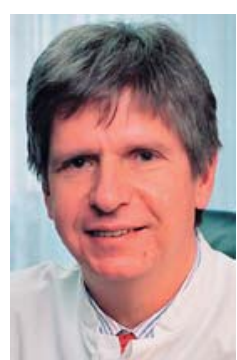

Dr. med. Clemens Fahrig
Chefarzt der Inneren Abteilung und Ärztlicher Direktor des Evangelischen Krankenhaus $\mathrm{Hu}-$ bertus in Berlin sowie Leiter des Gefäßzentrums Berlin-Brandenburg, kann dies nur bestätigen: „Gefäßsport ist eine sehr wirkungsvolle Therapie bei Oberschenkelarterienverschlüssen, um die Kollateralisierung der Beinarterien in Schwung zu bringen. Aber es geht nicht nur um die Kollateralen; die Bewegungstherapie hat eine Vielzahl von positiven Effekten auf den gesamten Organismus: Sie verbessert die Sauerstoffutilisation, die kardiale Leistung, kräftigt die Muskulatur. Die Gelenke werden wieder beweglicher, die Koordination verbessert sich, und letztendlich führt dies zu einer Erhöhung der Lebensqualität.“

Angebot an Gefäßsport zu gering

Um allen infrage kommenden Patienten Gefäßsport anbieten zu können, wäre ein flächendeckendes Netz an Gefäßsportgruppen wünschenswert. Dörr hat Anfang des Jahres eine ambulante Gefäßsportgruppe für die Region Barnim und Uckermark gegründet - eine Seltenheit 
im Bundesland Brandenburg. „Von einem flächendeckenden Netz sind wir weit entfernt“, bestätigt Fahrig. „Selbst in Berlin gibt es Stadtteile ohne eine einzige Gefäßsportgruppe, z.B. im Südwesten und Nordosten der Hauptstadt. In vielen anderen Großstädten existiert kein einziges Angebot. In ländlichen Gebieten sind Gefäßsportgruppen wegen der langen Anfahrtswege schwer durchführbar. Wir versuchen allerdings auch für diese Patienten etwas zu tun, indem wir ihnen Taktmeter mit ihrer individuellen Schrittfrequenz und ein Übungsprogramm mit nach Hause geben." In Berlin hat Fahrig gute Erfahrungen damit gemacht, auf die Sportvereine zuzugehen. Diese hätten ein Interesse, solche Gruppen anzubieten, da sie auch Fördermittel dafür bekommen können. „Mittlerweile gibt es berlinweit in verschiedenen Sportvereinen ca. 500 Gefäßsportler“, so Fahrig. Für sie wird der Gefäßsport von den Krankenkassen entweder komplett oder teilweise bezuschusst. In einem Berliner Sportverein bezahlt der Patient beispielsweise für die Teilnahme an der Gefäßsportgruppe 28 Euro, davon bekommt er bei regelmäßiger Teilnahme fast 20 Euro von seiner Krankenkasse zurück.

Um in der Wolletzer Gefäßsportgruppe mitmachen zu können, empfiehlt Dörr ihren Patienten, sich an den Hausarzt zu wenden, der für die Krankenkasse einen Antrag auf Kostenübernahme ausfüllt. Je nach Stadium der Krankheit bezahlt die Kasse bis zu zwei Jahren Reha-Sport, bei Bedarf auch länger

\section{„Reparaturmedizin“ hat Vorrang}

Bei derart niedrigen Kosten verwundert die geringe Umsetzung der LeitlinienEmpfehlungen umso mehr. „Eine Stentoder Bypass-OP kostet die Krankenkasse mehrere Tausend Euro, und es fragt niemand danach, ob durch Gefäßsport dieser Eingriff vielleicht hätte verhindert werden können“, kritisiert Fahrig. „Das Problem ist, dass alle Krankenhäuser unter einem hohen Leistungsdruck stehen. Durch den Aufbau und die Betreuung von Gefäßsportgruppen wird man nicht reich und kann sich nicht finanzieren, im Gegenteil: Es kostet viel Arbeit und Engagement. Das ist aus meiner Sicht eine sehr gefährliche Entwicklung. Die Empfehlung zum Gefäßsport halte ich für eine der am meisten missachteten Leitlinienempfehlungen." Paradoxerweise schreite diese Entwicklung voran, obwohl die Härte der
Empfehlung zum Gefäßsport in den Leitlinien zunehme. „Das ist ein eindeutiges Missverhältnis!“, stellt Fahrig fest. „In Berlin werden pro Jahr mehr als 15000 Gefäß-PTA durchgeführt, aber es sind nicht mal 1000 Gefäßsportler unterwegs. Dabei schließt eine Therapie die andere ja nicht aus. So ist beispielsweise im Beckenbereich eine Dilatation oder Operation unumgänglich, weil sich dort keine Umgehungskreisläufe bilden können. Aber auch in der Sekundärprävention nach Stenteinsatz oder Operation sollte dem Patienten Gefäßsport angeboten werden.“

\section{Ausschlusskriterien für den Gefäßsport}

Doch nicht alle Patienten mit Durchblutungsstörungen der Beine können mit Gefäßsport therapiert werden: Zur Behandlung der kritischen Ischämie (Fontaine-Stadien III und IV) ist Gehtraining nicht geeignet. Auch Patienten mit Beckenarterienstenosen und -verschlüssen sowie Stenosen der A. profunda femoris und Verschlüssen der ipsilateralen A. femoris superficialis profitieren nicht. Laut S3-Leitlinie sollte in diesen Fällen zunächst eine Gefäßrekanalisation erfolgen, bevor ein Gehtraining im Anschluss als Basisbehandlung eingeleitet wird. Auch orthopädische und/oder neurologische Begleiterkrankungen oder kardiopulmonale Funktionsdefizite können das Gehtraining erschweren, hier wird ebenfalls eine Abklärung empfohlen. „Besonders sorgfältig müssen auch Diabetiker untersucht werden, da sie wegen ihrer Polyneuropathie ein geringeres Schmerzempfinden haben. Bestehen beispielsweise bei einem Diabetiker im Stadium II Zweifel, ob dieses Stadium tatsächlich vorliegt, führen wir als Zusatzuntersuchung eine transkutane Sauerstoffpartialdruckmessung durch, mit der man eine kritische Ischämie sicher ausschließen kann“, berichtet Fahrig.

\section{Motivation der Ärzte steigern}

Ärgerlich findet Fahrig die Aussage, dass die Motivation vieler Patienten zum Gefäßsport gering sei. „Es liegt vor allem an der Motivation der Ärzte! Wir sagen dem Patienten: Sie können dem Ballon oder dem Bypass weglaufen! Wenn man den Patienten alle Zusammenhänge genau erklärt, ihnen verdeutlicht, dass immer noch operiert oder dilatiert werden kann, wenn der Gefäßsport nicht ausreicht dann sind mehr als $50 \%$ von ihnen zum
Training bereit. Einen Flyer in die Hand drücken reicht nicht!" Wichtig ist nach Fahrigs Ansicht auch, auf eine Altershomogenität der Gruppe zu achten, da die soziale Komponente eine Rolle spielt: „Wenn man das alles beachtet, wenn eine Gefäßsportgruppe so gut strukturiert und durchdacht ist wie ein Dilatation oder eine OP, dann wird der Erfolg nicht ausbleiben!“

Claudia Bruhn, Schmölln DOI 10.1055/s-0032-1330182 www.thieme.de/dmw 